\title{
Management of unstable angina
}

Recent studies show that invasive treatment given within the first week of onset of unstable angina reduces death and myocardial infarction incidence in moderate-risk and high-risk patients. There is better symptom relief and less readmission to hospital compared to a non-invasive approach. Most of the patients managed non-invasively have needed invasive procedures and revascularisation within 6 months. The evidence favours performing coronary angiography in every patient and where appropriate, doing a revascularisation procedure. When facilities for revascularisation are not readily available a method of risk stratification could be used to identify those needing urgent surgery from those in whom the procedures could be delayed. Those awaiting angiography could be managed with heparin, antiplatelet drugs and antianginal drugs. Angioplasty and bypass surgery could be considered for patients who are refractory to conservative treatment and who have multivessel disease. Hence, conservative and invasive management strategies have complementary rather than competing roles in the management of patients with unstable angina. The treatment could be tailored to patients' needs and treatment facilities available. Lancet 1999; 354: 694-5 and 701-7. In Sri Lanka the state hospitals will not be able to offer these facilities to the vast majority of patients. Most of the patients will have to be managed conservatively.

\section{Mistakes with methotrexate}

A report from Australia highlights the occurrence of serious side effects (eg. neutropaenia, hepatotoxicity, marrow depression) resulting from errors in dose and dose regimes of methotrexate (MTX). One source of error is the similar appearance of the $2.5 \mathrm{mg}$ and $10 \mathrm{mg}$ tablets of MTX. The other source is problems in communication. When patients are told to take a dose once a week, there are instances in which this dose is taken daily or on several days a week. Prescribers and pharmacists are advised to identify the tablet strength with the patient. When once a week regimes are given, it is recommended to specify the day on which this tablet should be taken. Australian Adverse Drug Reaction Bulletin 1999; 18: 14. As adverse drug monitoring is still in its infancy in Sri Lanka we have no idea about the extent of this type of mistake. However, as MTX is a widely used drug, both prescribers and pharmacists should pay attention.

\section{British guidelines for management of hypertension}

A summary of The British Hypertension Society guidelines for management of hypertension published in 1999 are given below. Details could be obtained from Journal of Human Hypertension. 1999; 13: 569-92.

4 All adults should have their blood pressure (BP) measured routinely at least every five years until the age of 80 years. Those with high normal values (135-139/85-89 $\mathrm{mm} \mathrm{Hg}$ ) and those who have high readings at anytime previously should have their BP re-measured annually.

Use non-pharmacological measures in all hypertensive and borderline hypertensive people.

- Initiate antihypertensive drug treatment in people with sustained systolic BP > 160 or sustained diastolic BP > 100 $\mathrm{mmHg}$.

Decide on treatment in people with sustained systolic BP between 140 and 159 or sustained diastolic BP between 90 and $99 \mathrm{~mm} \mathrm{Hg}$ according to the presence or absence of target organ damage, cardiovascular disease, diabetes or coronary heart disease risk.

* The optimal treatment targets are systolic BP < $140 \mathrm{~mm} \mathrm{Hg}$ : and diastolic BP < $85 \mathrm{~mm} \mathrm{Hg}$. The minimum acceptable level of control recommended is $<150 / 90 \mathrm{~mm} \mathrm{Hg}$.

$\leftrightarrow$ In the absence of contra-indications or compelling indications for other antihypertensive agents, low dose thiazide diuretics or beta blockers are preferred as first line treatment for the majority of hypertensive people.

Other drugs that reduce cardiovascular risk, such as aspirin and statins, must also be considered. 


\section{From the journals}

\section{Adrenaline in acute anaphylaxis}

The United Kingdom Resuscitation Council has published guidelines for the emergency treatment of acute anaphylactic reactions. These could be used in general practice, emergency departments and in other settings. Adrenaline (epinephrine) $1 / 1000$ solution is recommended, to be used by the intramuscular route. It could be repeated within five minutes if there is no improvement or the patient's condition deteriorates.

Solutions of adrenaline in strengths of $1 / 10000$ or $1 / 100000$ could be given slowly intravenously for patients in profound shock. If the intravenous route is to be used the patient should have electrocardiographic monitoring. The $1 / 1000$ solution of adrenaline should not be used intravenously.

Adrenaline by the subcutaneous route is also not recommended. It is important to give sufficient amounts of intravenous fluids, and cystalloids are preferred for this. British Medical Journal 1999; 319: 1-2.

\section{Risk of stroke reduced by fruit and vegetable intake}

A recent publication of data from 75596 women followed up for 14 years and 38683 men followed up for 8 years has shown that daily consumption of about five portions of fruits and vegetables reduces the risk of ischaemic stroke. Journal of the American Medical Association 1999; 282: 1233-9.

\section{Gestational diabetes}

Women with gestational diabetes have a 17 to $63 \%$ risk of nongestational diabetes within 5 to 16 years after the index pregnancy. The risk of diabetes is particularly high in women who have marked hyperglycaemia during or soon after pregnancy, who are obese and in whom the diabetes was diagnosed before 24 weeks of gestation. Women with a history of gestational diabetes should use an effective contraceptive method to minimise chances of becoming pregnant with untreated hyperglycaemia. Low dose combined oral contraceptive or copper-medicated intra-uterine contraceptive devices could be used during this period. New England Journal of Medicine 1999; 341: 1749-56.

\section{Bed rest: the benefit or harm should be evaluated by clinical trials}

A group of investigators have recently looked at trials which studied the effect of bed rest versus early mobilisation for medical conditions and procedures. Between January 1966 and June 1998 they found 39 randomised controlled trials which satisfied their entry criteria. In 24 trials investigating bed rest following a medical procedure, no outcomes improved significantly and in 8 the outcomes worsened significantly in some procedures (lumbar puncture, spinal anaesthesia, radiculography and cardiac catheterisation). In 15 trials investigating bed rest as a primary treatment, no outcomes improved significantly and in 9 the outcomes worsened significantly for some conditions (acute low back pain, labour, proteinuric hypertension during pregnancy, myocardial infarction and acute infectious hepatitis). These results suggest that the published trials give little support for bed rest as a form of management in a wide range of settings, and suggest that it may actually delay recovery and even harm the patient. The investigators conclude that we must not assume efficacy of bed rest. We should conduct further studies to determine the benefit or harm of bed rest as we do for other treatments. Lancet 1999; 354: 1229-33.

\section{R. L. Jayakody, Senior Lecturer in Pharmacology, University of Colombo.}

